\title{
Multi-country outbreak of Salmonella enteritidis infection linked to the international ice hockey tournament
}

\author{
T. PÄRN ${ }^{1,2} *$, V. DAHL ${ }^{2,3}$, T. LIENEMANN ${ }^{1}$, J. PEREVOSČIKOVS $^{4}$ AND \\ B. DE $\mathrm{JONG}^{5}$, on behalf of the International Outbreak Investigation Team $\dagger$ \\ ${ }^{1}$ Department of Infectious Diseases, National Institute for Health and Welfare (THL), Helsinki, Finland \\ ${ }^{2}$ European Programme for Intervention Epidemiology Training (EPIET), European Centre for Disease \\ Prevention and Control, (ECDC), Stockholm, Sweden \\ ${ }^{3}$ Public Health Agency of Sweden, Stockholm, Sweden \\ ${ }^{4}$ Department of Infectious Diseases Risk Analysis and Prevention, Centre for Disease Prevention and Control of \\ Latvia, Riga, Latvia \\ ${ }^{5}$ Surveillance and Response Support Unit, European Centre for Disease Prevention and Control, (ECDC), \\ Stockholm, Sweden
}

Received 19 February 2017; Final revision 14 May 2017; Accepted 22 May 2017;

first published online 14 June 2017

\section{SUMMARY}

In April 2015, Finnish public health authorities alerted European Union member states of a possible multi-country Salmonella enteritidis outbreak linked to an international youth ice-hockey tournament in Latvia. The European Centre for Disease Prevention and Control (ECDC), Finnish and Latvian authorities initiated an outbreak investigation to identify the source. The investigation included a description of the outbreak, retrospective cohort study, microbiological investigation and trace-back. We identified 154 suspected and 96 confirmed cases from seven countries. Consuming Bolognese sauce and salad at a specific event arena significantly increased the risk of illness. Isolates from Finnish, Swedish and Norwegian cases had an identical multiplelocus variable-number of tandem repeats analysis-profile (3-10-6-4-1). Breaches in hygiene and food storing practices in the specific arena's kitchen allowing for cross-contamination were identified. Riga Cup participants were recommended to follow good hand hygiene and consume only freshly cooked foods. This investigation demonstrated that the use of ECDC's Epidemic Intelligence Information System for Food- and Waterborne Diseases and Zoonoses platform was essential to progress the investigation by facilitating information exchange between countries. Cross-border data sharing to perform whole genome sequencing gave relevant information regarding the source of the outbreak.

Key words: International outbreak investigation, MLVA, Salmonella enteritidis PT1, whole genome sequencing (WGS).

\footnotetext{
* Author for correspondence: T. Pärn, Käbliku 1-33, Tallinn, Harjumaa 13426, Estonia. (Email: parntriin@gmail.com)

$\dagger$ Members of the international outbreak investigation team are listed in the end of the article.
}

\section{INTRODUCTION}

Salmonella is a bacterium that can cause salmonellosis: a gastrointestinal disease characterised by diarrhoea, fever, abdominal pain and vomiting. It is transmitted through contaminated and under-cooked food items, including eggs, poultry and meat products [1]. 
Salmonella is a common cause of outbreaks during mass-gatherings [2], mass caterings [3]. In addition, multi-country outbreaks have been previously reported [4]. During 2010-2012, the reported incidence of salmonellosis in the European Union and European Economic Area (EU/EEA) was $21 \cdot 5$ cases per 100000 and Salmonella enteritidis was the most frequently reported serovar [5]. The reported incidence of salmonellosis has been decreasing in the EU/EEA since 2006 [5] and this trend has been most accentuated for S. enteritidis [6].

On 14 April 2015, a cluster of six cases of Salmonella group D infection was identified in a hospital in Finland among players who had recently participated in the Riga Cup 2015, an international youth ice-hockey tournament in Latvia. The event consisted of five tournaments, which took place during the weekends from 27 March to 26 April 2015. Approximately 5000 participants (i.e. players, coaches and accompanying persons such as relatives) from 197 teams representing 19 countries attended the event. The Riga Cup 2015 matches took place at four arenas and lunches and dinners were served at venues for the participants (including coaches and accompanying persons). Participants were accommodated in seven different hotels where they had breakfast [7]. Because of the potential for an international outbreak, the Finnish National Institute for Health and Welfare (THL) notified European Centre for Disease Prevention and Control (ECDC) and other European countries on 16 April about a possible outbreak via the Epidemic Intelligence Information System for Food- and Waterborne Diseases (EPIS-FWD) [8].

Following the rapid risk assessment [8], additional cases, from other tournament weekends, were identified in Finland suggesting that the outbreak was still ongoing. On 24 April, the ECDC together with the Finnish and Latvian public health institutes initiated an international outbreak investigation to identify its extent and source and prevent further cases.

\section{METHODS}

\section{Descriptive epidemiology}

For case finding, we defined a suspected case as a Riga Cup 2015 participant with stomach cramps and/or diarrhoea and/or vomiting during or within 1 week after their stay in Riga. We defined a confirmed case as a Riga Cup 2015 participant with a laboratory confirmed Salmonella sp. infection with symptom onset during or within 1 week after their stay in Riga.

To find the cases, we acquired each participating team's contact person's e-mail from the organisers of Riga Cup 2015. On 29 April, the Latvian public health authorities disseminated the information regarding the outbreak via e-mail to the team leaders (excluding teams from Finland and Norway who had been already contacted by their respective national institutes). They asked team leaders to report the number of persons who met the suspected case definition in their teams. A reminder was sent on 2 May.

In Finland, where the outbreak was detected and case finding was performed by the Finnish national public health authority, different case definition was used. They identified the confirmed Salmonella sp. infection cases with travel history to Latvia from the national infectious disease register and by crosschecking these names with the Riga Cup 2015 attendee list [7]. Suspected cases were identified through an online survey. A Riga Cup participant having an onset of diarrhoea on or after 27 March qualified as a suspected case in Finland [7]. The outbreak team at the Norwegian Institute of Public Health also used the Norwegian Surveillance System for Communicable Diseases (MSIS) to identify the confirmed cases and also contacted the team leaders to find suspected cases. Both countries then reported the case numbers to the international outbreak investigation team.

\section{Analytical epidemiology}

For identifying a vehicle of infection, we conducted a retrospective cohort study. Riga Cup 2015 participants from the fourth tournament weekend (17-19 April), who had had their meals in the event arenas were invited to participate. A questionnaire was developed in English to collect information on demographic characteristics, symptoms and food history; the latter included which meals were eaten at what arena and which specific food items were consumed at each meal. We acquired the lunch and dinner menus from the event organisers and constructed the questionnaire according to the foods served. National authorities translated the questionnaire into their respective languages and sent it to the ice-hockey team leaders who then distributed it to the team members, either by e-mail or on paper. We developed a codebook for uniform data entry between the countries, the national agencies did the data entry in their 
country, and we then pooled the data. For the cohort study we defined a case as a Riga Cup 2015 participant with a laboratory-confirmed Salmonella group D or O:9 infection or a person with stomach cramps and/or diarrhoea and/or vomiting with the symptom onset starting 17 April and no later than a week after leaving the Riga Cup 2015.

\section{Data analysis}

We calculated total number of cases, number of teams affected and number of cases per country and also weekly attack rates (ARs) among the teams that had their meals in the arenas. To explore the associations between food items and illness, we calculated relative risks (RRs) with 95\% confidence intervals (CIs). The analyses were performed only for food items that were served during meals where $\geqslant 10$ cases had attended. Where several food items served at the same meal were significantly associated with illness in univariate analyses, we included them in a multivariable model to adjust for confounding. Generalized linear model was used to calculate risk ratios and $P$-values, which were considered significant if they were $<0 \cdot 05$. All analyses were performed using STATA Data Analysis and Statistical Software version 12 (Stata Corporation, College Station, TX, USA).

\section{Microbiological investigation}

Between 7 and 27 April, National Public Health authorities from Finland, Sweden and Norway cross-checked Salmonella sp. isolates that were notified to respective national registers with Riga Cup participant lists, in order to identify positive cases. Salmonella isolates were analysed by serotyping [9], phage typing [10] and multiple-locus variable-number of tandem repeats analysis (MLVA) [11]. In MLVA, the following loci nomenclature and order was used: SENTR7-SENTR5SENTR6-SENTR4-SE3. In order to compare and confirm the genetic relationship of Finnish and Swedish outbreak-related isolates with an identical MLVA type, five Finnish and three Swedish isolates were randomly selected and subjected to whole genome sequencing (WGS) by gene-by-gene analysis.

In order to study how common the detected Salmonella type is in Latvia, epidemiologists in Latvian institutes purposively sampled a selection of human and routine food quality-control Salmonella enteritidis isolates that were unrelated to the Riga Cup 2015 from the period during March-May 2015 for further investigation. These isolates were sent to THL in Finland for phage typing and MLVA analysis and those Latvian strains with identical phage type (PT) and MLVA type compared with the outbreak strain were also characterised using WGS in THL.

The genomic DNA for the WGS was isolated according to the instructions of MaqAttract ${ }^{\circledR}$ kit (Qiagen, UK). DNA quantity and quality was analysed using a gel electrophoresis and the Qubit ${ }^{\mathbb{R}}$ quantification platform (Invitrogen, USA). Approximately $0.2 \mathrm{ng} / \mu \mathrm{l}$ of DNA from each isolate was used for a pair-end library preparation (Nextera XT DNA Library Preparation Kit, Illumina, San Diego, CA, USA). Sequencing of the strains was performed with a MiSeq benchtop sequencer (Illumina, San Diego, CA, USA). The theoretical depth of coverage was calculated as a 100-fold. The schema for an ad hoc gene-by-gene analysis was defined based on $48 \mathrm{~S}$. enteritidis complete genomes available at Genbank using a target definer tool within the Ridom SeqSphere+ software (Ridom, Münster, Germany). The sequence paired-end reads of Finnish, Swedish and Latvian strains were de novo assembled to contigs using SPAdes genome assembler algorithm (http:// bioinf.spbau.ru/spades) [12]. The imported sequence reads were trimmed and the adapters of Nextera library were removed. The genomes of Swedish and Latvian isolates were compared with Finnish outbreak-related and non-outbreak related isolates using the gene-by-gene analysis. The Finnish nonoutbreak isolates chosen for reference represented the most common $S$. enteritidis PTs in Finland and one of these had the same PT and a similar MLVA type (one locus difference) as the outbreak strain. Based on genome sequence data, multilocus sequence type (MLST) of seven house-keeping genes and 3648 core genome target genes were identified. Each of the target genes have an assigned allele number and if the target gene was found and was different from the reference genome or any other queried genome a new allelic number was assigned to that locus. For comparison purposes, the allelic differences of the studied strains were shown as a minimum spanning tree (MST) that was performed using tools within the Ridom SeqSphere+ software.

\section{Environmental investigation and trace-back}

Initially two event arenas were suspected as the source of the outbreak. Information based on the interviews with the first Finnish cluster pointed to arena A and 
an Estonian team with five suspected cases pointed to arena B [7]. On 16 April, an environmental investigation was performed in the canteen kitchen in arena $\mathrm{A}$ by specialists from the Centre for Disease Prevention and Control of Latvia and the Latvian Food and Veterinary Service. Follow up investigations were performed on 24 and 27 April. The canteen kitchen in arena B was investigated on 29 April. The specialists collected environmental, food swabs and stool samples from the canteen staff from arena A and arena B. All the samples were tested for Salmonella by culturing. The Latvian Food and Veterinary services reviewed the purchase receipts from the restaurants at arena A and B to identify the producers, importers and the suppliers of the suspected food items (i.e. meat products and products that did not require heating before serving).

\section{RESULTS}

\section{Descriptive epidemiology}

We received replies from $85 \%$ (168/197) of the teams, which represent $83 \%(4391 / 5290)$ of the total participants. Team leaders and national public health authorities reported a total of 250 cases (154 suspected and 96 confirmed) from 48 teams (Figure 1). The AR over the course of five tournament weekends was $5 \%$ (250/5290). Cases came from: Finland (57 suspected, 81 confirmed), Sweden (56 suspected, 14 confirmed), United Kingdom (15 suspected), Norway (13 suspected, 1 confirmed), Estonia (5 suspected), Latvia (4 suspected) and Lithuania (4 suspected). During the five tournament weekends, 147 teams had their meals in at least one of the four event arenas. The rest of the teams were from Latvia and they provided their own meals. The weekly team AR was the highest during the fourth tournament weekend (16/29: AR = $55 \%)$ and lowest during the fifth tournament weekend $(2 / 33: \mathrm{AR}=6 \%)$.

\section{Analytical epidemiology}

On the fourth tournament weekend 29 teams had their meals in the event arenas. Of these, 15 teams from four countries (Finland $(n=7)$, Sweden $(n=6)$, Latvia $(n=1)$, United Kingdom $(n=1))$, participated in the cohort study. Of the 207 responders, 57 were cases. About half of the cases were $<15$ years old $(53 \%)$ and hockey players (51\%). Median duration of the illness was 5 days (range 1-19). Date of symptom onset was available for 54 of the 57 cases (Figure 2). Of the 12 cases with symptom onset during the first and second day of the fourth tournament weekend, nine had taken all their catered meals before symptom onset in arena A.

Of the 20 meals served in the four arenas during the $17-19$ April, eight could explain $\geqslant 10$ cases. These included all five meals served in arena $\mathrm{A}$, two meals served in arena $C$ (17 April) and one meal in arena B (19 April). Lunch served in arena A on 17 April could explain 34 of the 57 cases, which was the highest number of cases explained by a single meal. None of the food items served in arena B or C were significantly associated with being a case. In univariate analyses, participants who consumed Bolognese sauce or salad in arena A had significantly higher risk of becoming ill compared with those who did not consume these food items (Table 1). Having eaten at least one of these food items (i.e. either salad or Bolognese sauce or both of them) were mentioned by 33/57 cases. After inclusion of Bolognese sauce and salad served on the 17 April lunch in the multivariable model, both exposures remained significant: Bolognese sauce: $\mathrm{RR}=3 \cdot 4,95 \%$ CI $1 \cdot 5-7 \cdot 7, \quad P=$ 0.004; salad: $\mathrm{RR}=3 \cdot 4,95 \%$ CI $1 \cdot 3-9 \cdot 8, P=0 \cdot 021$.

\section{Microbiological investigation}

The Swedish $(n=14)$, Finnish $(n=14)$ and Norwegian $(n=1)$ human faecal isolates typed during the outbreak were characterised as $S$. enterica serovar Enteritidis PT1 with MLVA type 3-10-6-4-1. Five Finnish and three Swedish outbreak-related isolates were characterised using an ad hoc WGS protocol at THL. These had identical MLST type ST11 and showed 21-48 allelic differences to each other in MST using the results of 3648 core genome genes by gene-by-gene analysis. These outbreak-related isolates differed from the Finnish $S$. enteritidis isolates from cases without links to the Riga Cup outbreak (Figure 3).

Six human and four routine food own-control (two isolates from broiler, one from minced pork, one from chicken skin) Latvian isolates that were unrelated to the Riga Cup were sent to THL for further characterisation. Of these, four human and the minced pork isolate were characterised as $S$. enteritidis PT1 with identical MLVA profile (3-10-6-4-1) and MLST type (ST11) to the outbreak strain. According to the gene-by-gene analysis three isolates of human origin were similar to the Swedish and Finnish outbreak 


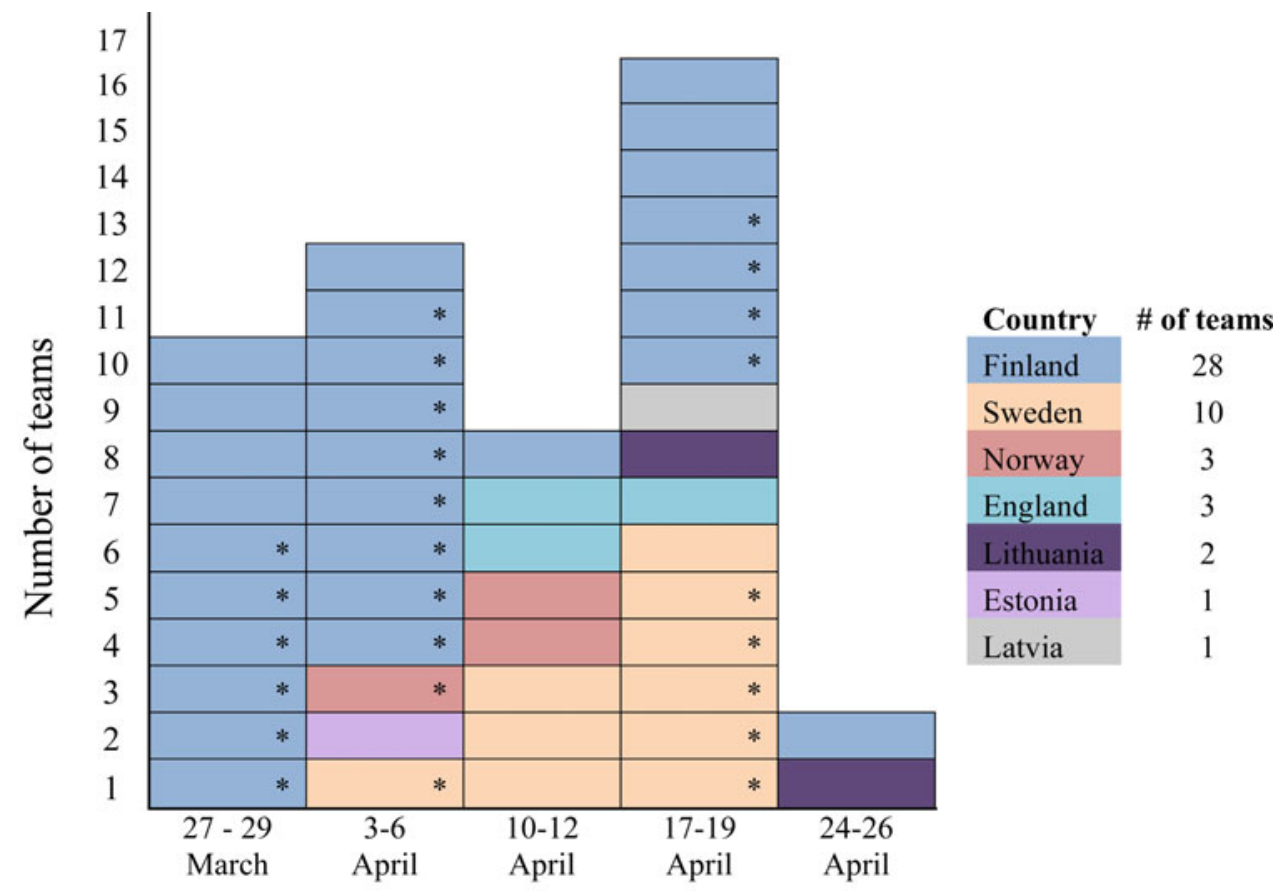

Tournament weekend

*indicates the team with a confirmed case

** total number of participating teams was 197

Figure 1. Distribution over time of teams with suspected and/or confirmed Salmonella sp. infection cases, by country and Riga Cup tournament weekend, Latvia, March-April $2015(n=48)$

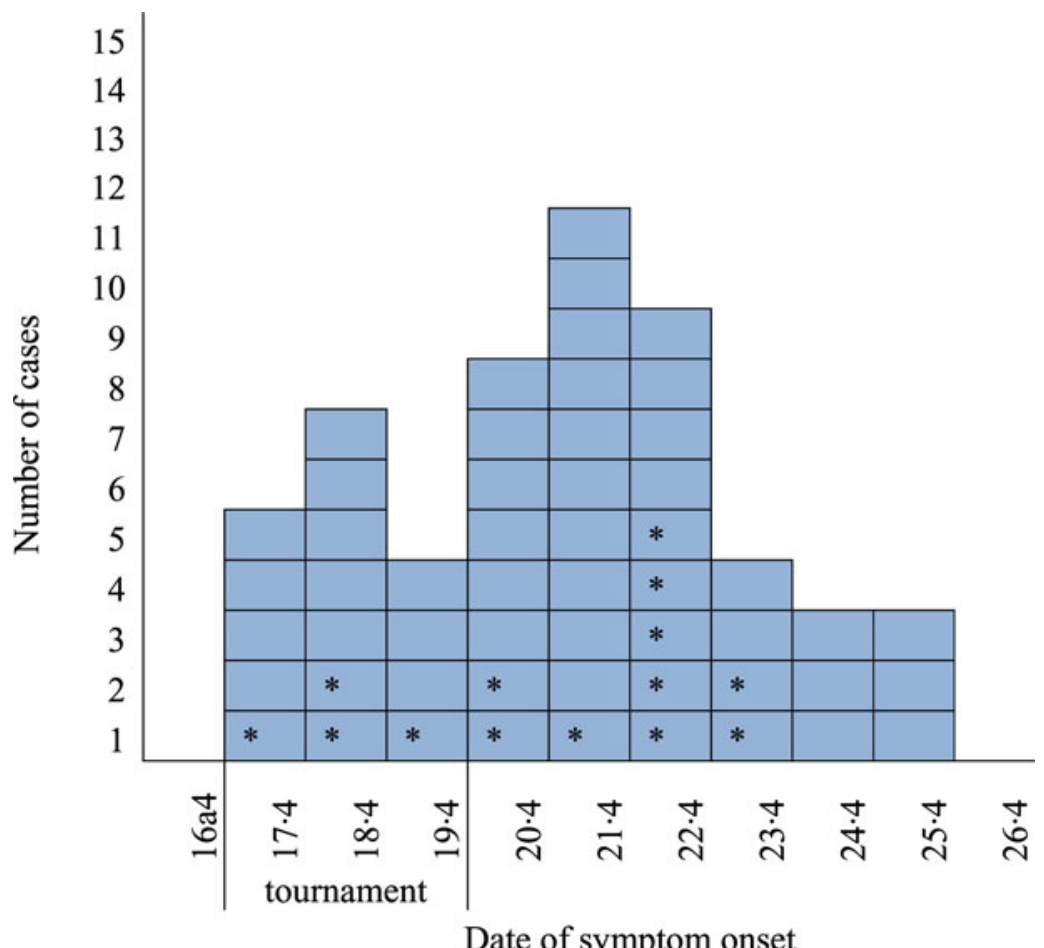

*confirmed Salmonella sp.

Figure 2. Cases of Salmonella sp. infection by date of symptom onset among cohort study participants, Riga Cup, Latvia, April, $2015(n=54)$ 
Table 1. Food exposures significantly increasing the risk of illness in univariate analysis during Salmonella outbreak, Riga Cup, Latvia, 2015

\begin{tabular}{|c|c|c|c|c|c|c|c|c|c|}
\hline \multirow[b]{2}{*}{ Arena A } & & \multicolumn{3}{|l|}{ Exposed } & \multicolumn{3}{|c|}{ Unexposed } & \multirow[b]{2}{*}{$\mathrm{RR}$} & \multirow[b]{2}{*}{$95 \% \mathrm{CI}$} \\
\hline & & Cases, $n$ & Total, $n$ & AR, $\%$ & Cases, $n$ & Total, $n$ & AR, $\%$ & & \\
\hline \multirow[t]{6}{*}{17 April } & Lunch & & & & & & & & \\
\hline & Spaghetti & 24 & 57 & 42 & 5 & 28 & 18 & $2 \cdot 4$ & $1 \cdot 0-5 \cdot 5$ \\
\hline & Bolognese sauce & 23 & 51 & 45 & 5 & 36 & 14 & $3 \cdot 2$ & $1 \cdot 4-7 \cdot 7$ \\
\hline & Salad & 26 & 56 & 46 & 4 & 27 & 15 & $3 \cdot 1$ & $1 \cdot 2-8 \cdot 1$ \\
\hline & Dinner & & & & & & & & \\
\hline & Salad & 17 & 28 & 61 & 3 & 17 & 18 & $3 \cdot 4$ & $1 \cdot 2-10 \cdot 0$ \\
\hline \multirow[t]{2}{*}{18 April } & Lunch & & & & & & & & \\
\hline & Salad & 20 & 29 & 69 & 4 & 21 & 19 & $3 \cdot 6$ & $1 \cdot 5-9 \cdot 0$ \\
\hline \multirow[t]{2}{*}{19 April } & Lunch & & & & & & & & \\
\hline & Salad & 12 & 26 & 46 & 5 & 27 & 18 & $2 \cdot 5$ & $1 \cdot 0-6 \cdot 1$ \\
\hline
\end{tabular}

$\mathrm{AR}$, attack rate; $\mathrm{RR}$, relative risk; $\mathrm{CI}$, confidence interval.

strain but the minced pork meat was excluded from the cluster (Figure 3).

\section{Environmental investigation and trace back}

In both investigated arenas, the meals were prepared in the kitchen on-site. Raw products were bought 1-2 days before the tournament and all leftovers were discarded in the evenings. Food and Veterinary Services of Latvia found the following noncompliances to Latvian food safety regulations in the catering establishment in arena A: (1) uncovered raw meat was kept in the same refrigerator as ready to eat meat products; (2) non-compliances in internal food traceability procedures (i.e. no time labelling for products that were frozen on site); and (3) dirty container for small cooking equipment.

All the samples collected from the kitchens $(n=41)$, from the food $(n=14)$ and from the staff $(n=12)$ were negative for Salmonella sp.

The Bolognese sauce served on 17 April lunch contained minced pork originating from Latvia. Several ingredients were used in the salads: Chinese cabbage (Poland), cucumbers (Poland), carrots, onions (Lithuania), tomatoes, iceberg lettuce (Spain), radish (Italy, the Netherlands), cabbage (Latvia), and salad dressing (Latvia).

\section{Control measures}

Following the initial investigation in arena $\mathrm{A}$ on 16 April, the Latvian Food and Veterinary service requested the food business operator to improve the hygiene in the kitchen. Additional control measures were implemented and are described previously [7]. In addition, participants of the Riga Cup 2015 and of other ice hockey events that took place in arena A were informed about the ongoing outbreak. They were recommended to follow good hand hygiene practices and consume only heat-treated foods.

\section{DISCUSSION}

We investigated a multi-country $S$. enteritidis outbreak among participants in a junior ice hockey tournament in Latvia 2015. Our investigation identified that cases occurred in association with different tournament weekends and that only consumption of food items served in arena A were statistically significantly associated with the illness. It suggests that a continuous point source in arena A caused this outbreak. Our hypothesis of a common source is further strengthened by the MLVA and WGS results, which provided a strong link between the cases from three of the affected countries. Hence, it is unlikely that cases got infection on their way to the tournament or from the foods served in the hotel as different means of transportations and hotels were used by cases.

Poor hygiene has been observed among food providers in large-scale events [2]. It is also well known that inadequate hand hygiene and rise in food production facilitate the transmission of bacteria and crosscontamination [2]. Latvian Food and Veterinary services identified breaches in hygiene and food storing practices in the kitchen in arena A. This might have led to the contamination of the food items served to the participants. Cases occurred over several weekends, indicating a continuously present source in 

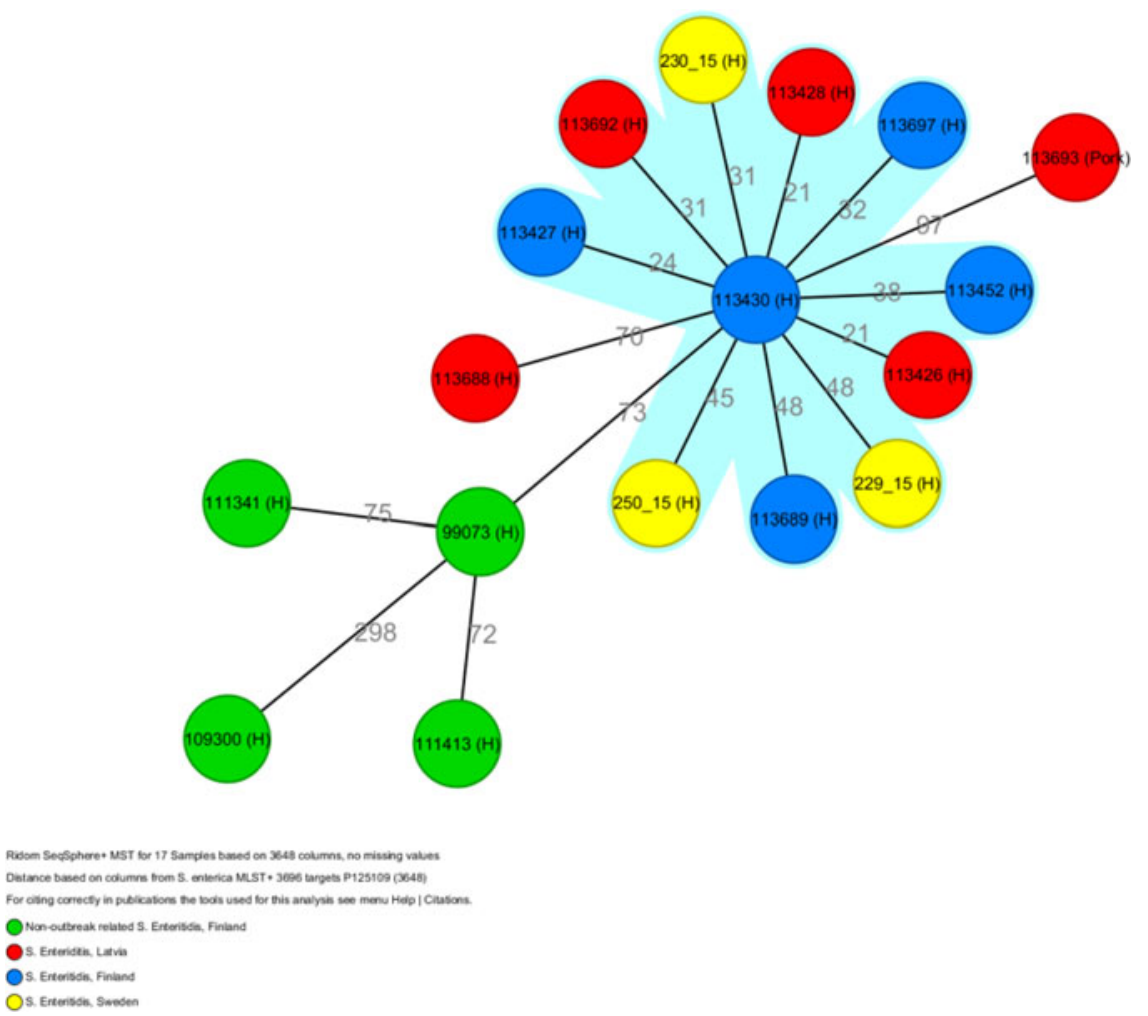

Figure 3. Minimum spanning tree of Finnish (5 human, blue), Swedish (3 human, yellow), 5 Latvian (4 human, 1 minced pork, red) outbreak related isolates and Finnish (4 human, green) non-outbreak related isolates using gene-by-gene analysis, Riga Cup, Latvia, 2015.

arena A. According to the kitchen staff, all the food items except spices were purchased only 1-2 days before each tournament weekend. Salmonella has been previously identified as the causative pathogen in several reported outbreaks that were linked to the consumption of spices [13]. Microbiological investigation was not performed on spices in this investigation.

The results of our cohort study suggest that during the Riga Cup 2015 fourth weekend the cases acquired infection via Bolognese sauce or salad served in arena A. Five cases had symptom onset already on the first tournament day, which probably refer to a high-dose ingestion during the meals. All these five cases had eaten salad during the lunch in arena A. As Salmonella incubation period ranges from 6 to $72 \mathrm{~h}$, there is also a possibility that the cases were infected before the tournament. This, however, is unlikely as the cases were from Latvia and Sweden and prior to the tournament they had no common food exposure. Bolognese sauce and salad were often consumed together making the identification of the contaminated food item difficult. As preparation of the Bolognese sauce requires boiling and Salmonella is eliminated when heat-treated at $70{ }^{\circ} \mathrm{C}[1]$, it is more likely that the bacteria were transmitted via ingredients in the salad which was served as raw. Salad could have been cross-contaminated in the kitchen when handling its ingredients after usage of the contaminated food product (e.g. meat) without hand-washing. We have no microbiological evidence to support the hypothesised cross-contamination, as none of the food items served during the tournament weekends were available for testing and Salmonella was not detected in any of the environmental swabs. We also cannot rule out that the contamination of the salad ingredients occurred in the setting where it was grown and harvested or processed. As the outbreak strain seems to be circulating in Latvia, it could be that manure used for growing the vegetables contaminated the cabbage (the only salad ingredient originating from Latvia). We did not perform any environmental investigation on the premises of the cabbage producers.

The lowest AR among the teams was observed during the last Riga Cup 2015 weekend, with no confirmed cases, indicating that recommendations given to the participants to consume only freshly cooked food and thereby exclude the salad was 
effective. The decrease in the number of cases could also be attributed to the recommendations given to improve the hygiene in the kitchen in arena $\mathrm{A}$.

Microbiological methods are an important part of the outbreak investigation to support epidemiological findings and/or to detect the outbreaks [4]. We used several methods, including phage typing, MLVA and WGS to characterise the isolates. The results by all methods used confirmed that the cases from different countries were infected with an indistinguishable strain. Isolates from three non-outbreak-related Latvian human cases that clustered with the outbreakrelated strains from Finland and Sweden based on WGS results indicate this strain might be circulating currently in Latvia. Improper food handling, transportation, poor storage, cross-contamination at a packaging or shipping facility, etc can give rise to further cases occurring. Interview of these sporadic Latvian cases failed to identify a common food exposure.

WGS is a rapid way to provide highly discriminating information on pathogens [14]. It is increasingly applied in outbreak investigations [15] since it can generate full length sequences of pathogens in a short time which, with a high resolution, can determine the genetic similarity of different isolates found during an outbreak. A major limitation of WGS is the lack of validation studies and internationally harmonised WGS protocols. A model process on how to routinely analyse the data have been developed by an ECDC expert group on introduction of nextgeneration typing methods for surveillance of foodand waterborne diseases [16]. Due to the international scope of food trade and food-borne pathogens, the relevance of data sharing and cross-border collaboration is highlighted in their report [16].

Detection of outbreaks, especially international ones, can be complex if the cases are spread out over time, place and if isolates are not routinely typed. A cluster of Riga Cup 2015 participants with $S$. enteritidis infection that attended the same hospital in Finland facilitated the early identification of this outbreak. Substantial coordination with clear roles and responsibilities is needed in international outbreak investigations [17, 18]. ECDC's support by sending staff at short notice to Latvia and also ECDC's coordination in this outbreak was important for facilitating the investigation, because Latvia itself had no reported cases initially, and the outbreak was first detected outside the country, making hypothesis generation difficult in Latvia.
Timely communication and data sharing between epidemiologists, microbiologists and food safety authorities is challenging in international investigations. Regular teleconferences for information exchange and access to information sharing systems like EPIS-FWD for data sharing and data pooling between the affected countries are essential to progress in this type of investigations. As uploading information to EPIS-FWD is additional work for member states, clear guidance should be given to the countries on how, when and what data to upload.

There were several limitations in this investigation. First, we did not include in the questionnaire a question on whether the respondent had eaten the salad served in arena A during dinner on 18 April. Inclusion of this question might have resulted in stronger associations between the exposure to salad and illness. Omission of this question was due to the rapid implementation of the questionnaire without time for pilot testing. No follow-up questionnaire was distributed, as contacting the team leaders to distribute questionnaires once more would have been labour and time-intensive for an outbreak that had already ended. Also we would have introduced recall bias in the results as time had passed from the outbreak already. Second, some cases might have been double reported since ice hockey team leaders notified the aggregated number of suspected cases and national authorities reported individual data. This was most likely to have occurred for Swedish cases since Norwegian and Finnish public health institutes performed case finding by themselves, either by interviewing or via a survey. In addition, reliance of the suspected cases limits the reliability of the results. Third, we included only cases from the fourth tournament weekend in our cohort study, limiting the generalisability of the findings for all the Riga Cup 2015 weekends. Knowing that similar menus were used during the whole Riga Cup 2015 and salad was the only raw product served to participants, it is, however, likely that salad was also the infection vehicle during other tournament weekends. As known, the longer the time interval between the event and the assessment, the higher is the probability to incorrectly recall. Hence, including participants only from the fourth weekend limited the possibility of recall bias. Finally, only partial traceback was performed in this investigation due to limited resources. Details regarding the origin of the non-outbreak-related isolates and investigation in the food producers' premises might have given further insight to possible routes or points of contamination. 
In conclusion, we investigated a multi-country outbreak of Salmonella. We identified the most likely source to be salad served in arena A. Cross-contamination of the salad might have occurred in the kitchen of this particular arena. Riga Cup participants were recommended to follow good hand hygiene and consume only freshly cooked foods. Furthermore, this outbreak investigation demonstrated the benefits of EPIS-FWD during international investigations and we recommend countries to continue the use of EPIS-FWD during multi-country outbreak investigations as information sharing platform. Harmonising the protocol for WGS among EU countries would facilitate the comparison of results.

\section{MEMBERS OF THE INTERNATIONAL OUTBREAK INVESTIGATION TEAM}

Estonia (Health Board) K. Raska; Finland (National Institute for Health and Welfare) R. Rimhanen-Finne, S. Huusko, S. Salmenlinna, J. Halkilahti, J. Ollgren, AK. Pesola; Hungary (National Centre for Epidemiology) A. Feher; Latvia (Centre for Disease Prevention and Control of Latvia) I. Lucenko, R. Korotinska, I. Kantsone (EPIET), (Food and Veterinary Services of Latvia) T. Marcenkova, (Latvian Centre of Infectious Diseases) J. Storozenko; Lithuania (Vilnius Public Health centre) G. Aleksiene; Norway (Norwegian Institute of Public Health) H. Lange (EPIET), L. Vold; Sweden (Public Health Agency of Sweden) E. Löf, C. Jernberg, E. Alm; United Kingdom (Public Health England) A. Awofisayo; European Centre for Disease Prevention and Control (ECDC): C. Gossner, T. Westrell, T. Korhonen, N. Danielsson, J. Takkinen, T. Niskanen, M. Muehlen

\section{ACKNOWLEDGEMENTS}

The authors thank the employees of the national institutes involved in the outbreak investigation, WHO EURO (Ana Paula Coutinho, Hilde Kruse) and WHO INFOSAN (Carmen Savelli) for assisting the outbreak investigation.

\section{DECLARATION OF INTEREST}

None.

\section{REFERENCES}

1. Heymann DL. Control of Communicable Diseases Manual, 19th edn. Washington DC, USA: American Public Health Association, 2008.
2. Botelho-Nevers E, Gautret P. Outbreaks associated to large open air festivals, including music festivals, 1980 to 2012. Eurosurveillance 2013; 18: pii20426.

3. Osimani A, Aquilanti L, Clementi F. Salmonellosis associated with mass catering: a survey of European Union cases over a 15-year period. Epidemiology and Infection 2016; 18: 1-13.

4. Inns T, et al. A multi-country Salmonella enteritidis phage type $14 \mathrm{~b}$ outbreak associated with eggs from a German producer: 'near real-time' application of whole genome sequencing and food chain investigations, United Kingdom, May to September 2014. Eurosurveillance 2015; 20: pii:21098.

5. European Centre for Disease Prevention and Control (ECDC). 'Surveillance of seven priority food-and waterborne diseases in the EUIEEA 2010-2012. Stockholm: ECDC; 2015. Available from: http://ecdc.europa.eu/en/ publications/Publications/food-and-waterborne-diseasessurveillance-report-2015.pdf.

6. Frank C, et al. Marked decrease in reporting incidence of salmonellosis driven by lower rates of Salmonella enteritidis infections in Germany in 2008: a continuing trend. Eurosurveillance 2009; 14: pii:19154.

7. Pesola AK, et al. Multinational outbreak of Salmonella enteritidis infection during an international youth ice hockey competition in Riga, Latvia, preliminary report, March and April 2015. Eurosurveillance 2015; 20: pii:21133.

8. European Centre for Disease Prevention and Control (ECDC). Multinational Outbreak of Salmonella Enteritidis Infections among Junior Ice Hockey Players Attending the Riga Cup 2015-27 April 2015. Stockholm: ECDC; 2015. Available from: http://ecdc.europa.eu/en/publications/Publications/Salmonella-NorwayLatvia-Finland-rapid-risk-assessment.pdf.

9. Issenhuth-Jeanjean S, et al. Supplement 2008-2010 (no. $48)$ to the White-Kauffmann-Le Minor scheme. Research in Microbiology 2014; 165: 526-530.

10. Ward LR, de Sa JD, Rowe B. A phage-typing scheme for Salmonella enteritidis. Epidemiology and Infection 1987; 99: 291-294.

11. Hopkins KL, et al. Standardisation of multilocus variable-number tandem- repeat analysis (MLVA) for subtyping of Salmonella enterica serovar Enteritidis. Eurosurveillance 2011; 16: pii:19942.

12. Bankevich A, et al. SPAdes: a new genome assembly algorithm and its applications to single-cell sequencing. Journal of Computational Biology 2012; 19: 455-477.

13. Van Doren JM, et al. Foodborne illness outbreaks from microbial contaminants in spices, 1973-2010. Food Microbiology 2013; 36: 456-464.

14. Gilchrist CA, et al. Whole-genome sequencing in outbreak analysis. Clinical Microbiology Reviews 2015; 28: 541-563.

15. Le VT, Diep BA. Selected insights from application of whole genome sequencing for outbreak investigations. Current Opinion in Critical Care 2013; 19: 432-439.

16. European Centre for Disease Prevention and Control (ECDC). Expert Opinion on the Introduction of NextGeneration Typing Methods for Food-and Waterborne 
Diseases in the EU and EEA. Stockholm: ECDC; 2015.

Available from: http://ecdc.europa.eu/en/publications/ Publications/food-and-waterborne-diseases-next-generationtyping-methods.pdf.

17. Sane J, et al. Multistate foodborne hepatitis A outbreak among European tourists returning from Egypt - need for reinforced vaccination recommendations, November 2012 to April 2013. Eurosurveillance 2015; 20: pii:21018.

18. Ammon A, Tauxe RV. Investigation of multi-national foodborne outbreaks in Europe: some challenges remain. Epidemiology and Infection 2007; 135: 887-889. 